\title{
The efficacy of a low-fat diet to manage the symptoms of bile acid malabsorption - outcomes in patients previously treated for cancer
}

\author{
Authors: Amy Jackson, ${ }^{A}$ Amyn Lalji, ${ }^{\mathrm{B}}$ Mohammed Kabir, ${ }^{\mathrm{C}}$ Ann Muls, ${ }^{\mathrm{D}}$ Caroline Gee, ${ }^{\mathrm{E}}$ Susan Vyoral, ${ }^{\mathrm{F}}$ Clare Shaw ${ }^{\mathrm{G}}$ \\ and $\mathrm{H}$ Jervoise $\mathrm{N}$ Andreyev ${ }^{\mathrm{H}}$
}

Dietary fat ingestion triggers bile secretion into the gastrointestinal tract. Bile acid malabsorption affects $>1 \%$ of the population, causing loose stool and other gastrointestinal symptoms. The diagnosis is frequently missed. Treatments are often considered ineffective. We evaluated low-fat diets for managing gastrointestinal symptoms in these patients. All patients reporting type 6 or 7 stool were offered a selenium-75 homocholic acid taurine (SeHCAT) scan. Prospective data in patients with 7 -day scan retention $<20 \%$ were analysed. Patients requiring a bile acid sequestrant were given this before receiving dietary advice. Patients completed a 7-day food diary before dietetic consultations. Personalised dietary interventions, providing $20 \%$ of daily energy from fat, were prescribed. Symptoms were assessed using a modified gastrointestinal symptom rating scale questionnaire before and 4-12 weeks after dietary intervention. A total of 114 patients ( 49 male, median age 64 years, median body mass index $27 \mathrm{~kg} / \mathrm{m}^{2}$ ) were evaluated. $44 \%$ of these patients were taking colesevelam. After dietary intervention, there was statistically significant improvement in abdominal pain and nocturnal defecation ( $0.2 \%$ alpha, $p=0.001)$. Improvement in bowel frequency, urgency, flatulence, belching, borborygmi and stool consistency were seen, but did not reach statistical significance ( $p \leq 0.004-0.031)$. Dietary intervention is an effective treatment option for patients with symptomatic bile acid malabsorption and should be routinely considered.

Authors: Ateam navigator, GI unit, Royal Marsden NHS Foundation Trust, London and Sutton, UK; ${ }^{B}$ senior project manager, GI unit, Royal Marsden NHS Foundation Trust, London

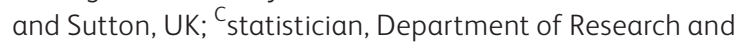
Development, Royal Marsden NHS Foundation Trust, London and Sutton, UK; ${ }^{D}$ Macmillan nurse consultant, GI unit, Royal Marsden NHS Foundation Trust, London and Sutton, UK; ${ }^{\text {S }}$ enior clinical nurse specialist, GI unit, Royal Marsden NHS Foundation Trust, London and Sutton, UK; F senior dietitian, Royal Marsden NHS Foundation Trust, London and Sutton, UK; ${ }^{\mathrm{G}}$ consultant dietitian, Royal Marsden NHS Foundation Trust, London and Sutton, UK; ${ }^{H}$ consultant gastroenterologist, GI unit, United Licolnshire Hospitals NHS Trust, Lincoln County Hospital, Lincoln, UK
KEYWORDS: Bile acid malabsorption, diarrhoea, gastrointestinal, low-fat diet, SeHCAT, sequestrant

\section{Introduction}

Bile acids are synthesised in the liver and stored in the gall bladder. Particularly, triglycerides containing long-chain fatty acids, long-chain free fatty acids, aromatic-aliphatic amino acids and intact proteins in food - but not carbohydrates stimulate entero-endocrine cells to secrete cholecystokinin, which stimulates contraction of the gall bladder and relaxation of the sphincter of Oddi, allowing secretion of bile acids into the duodenum. ${ }^{1}$ They play a key role in the emulsification and solubilisation of lipids in the small intestine, aiding their digestion and absorption. Lipids are absorbed in the small bowel and the bile acids are released. A small proportion of these bile acids are reabsorbed passively throughout the small bowel; however, the vast majority are reabsorbed over a relatively short section of the terminal ileum as a result of an active absorption mechanism. They enter the entero-hepatic circulation and are reused six to ten times each day. Bile acid production in the liver is regulated by fibroblast growth factor 19 (FGF19) secretion, which is produced in the terminal ileum. ${ }^{2}$ Approximately $5 \%$ of the circulating pool of bile acids is not absorbed and reaches the colon where deconjugation by intestinal bacteria converts them into secondary bile acids. These have a role in regulating normal bowel function. ${ }^{3} \mathrm{An}$ excessive volume of secondary bile acids in the colon stimulates electrolyte and water secretion, increases gastrointestinal (GI) motility, shortens colonic transit time and inhibits water and electrolyte reabsorption. This results in loose stool and potentially in a number of other symptoms, which can include urgency of defecation, abdominal bloating/cramps, flatulence, abdominal pain, steatorrhoea and nocturnal defecation. ${ }^{4-6}$ Constipation (following episodes of diarrhoea) is rated as the worst symptom in $20 \%$ of those affected in one study. ${ }^{4}$ Symptoms are often severe, for example, chronic episodic faecal incontinence in up to half of all patients. However, when individuals develop GI symptoms due to excessive bile acids reaching the colon, these reflect the amount of bile secreted many hours previously, so they can rarely see the association between their symptoms and their dietary intake of fat. 
Bile acid malabsorption (BAM) is defined as a defect in absorptive mechanisms in the terminal ileum because of ileal disease, mucosal dysfunction or surgical resection. Recently, the term bile acid diarrhoea (BAD) has been popularised. It describes a syndrome with that same wide range of symptoms (ie not just diarrhoea), but a wholly distinct causative mechanism. It is due to impaired FGF19 feedback inhibition by a non-diseased, intact ileum, resulting in excessive bile acid synthesis by the liver, overwhelming ileal absorptive capacity and resulting in excessive bile salts reaching the colon. ${ }^{7}$

There is no simple diagnostic test available for BAM/BAD worldwide. However, selenium-75 homocholic acid taurine (SeHCAT) scanning is available in 10 European countries, Canada and Australia (but not the USA) and has a sensitivity of $89-97 \%$ and a specificity of close to $100 \%$ for the diagnosis of BAM/BAD. ${ }^{8,9}$ Even where the SeHCAT scan is available, usage varies widely. Clinicians in the UK request 10 times as many scans per million population compared with any other country (personal communication, General Electric). In addition, while a SeHCAT scan result will indicate that malabsorption is occurring, it does not necessarily predict the variety or severity of patients' symptoms because these also depend on the amount of fat in the diet.

Studies using SeHCAT scanning consistently suggest that BAM/BAD affect large numbers of people. Primary BAD affects approximately $1 \%$ of the population. ${ }^{10}$ Secondary $\mathrm{BAM} / \mathrm{BAD}$ - described frequently after upper GI surgery, with pancreatic disease, during chemotherapy or biological therapies for cancer, during and after pelvic radiotherapy, after terminal ileal resection for Crohn's disease or cancer, in those with microscopic colitis and a number of other conditions ${ }^{11-20}$ - affects unquantified, but substantial, numbers of patients. The failure to diagnose this condition - it is frequently misdiagnosed as irritable bowel syndrome - not only means that difficult symptoms are not treated optimally but also impacts on the health economy; many undiagnosed patients undergo, sometimes repeatedly, unnecessary and expensive tests (eg colonoscopy) and are offered ineffective and frequently costly medications.

There are probably two main reasons why BAM/BAD remains underdiagnosed. First, many key opinion leaders in gastroenterology have no access to diagnostic tests for this condition, so have no objective markers to help them manage it; second, there is a widespread perception that treatment options are not very effective. However, there have been surprisingly few studies investigating and reporting outcomes for the various treatment options for $\mathrm{BAM} / \mathrm{BAD}$, let alone the use of low fat diets. ${ }^{6,21-23}$

The aim of this study was to evaluate the efficacy of lowfat dietary interventions in the management of bile acid malabsorption.

\section{Method}

This study was reviewed and approved as a service evaluation by the Royal Marsden Hospital Committee for Clinical Research and did not require informed consent from patients.

This was an evaluation of prospectively and systematically recorded data from consecutive patients with a 7-day SeHCAT scan result of $<20 \%$ at The Royal Marsden Hospital Foundation Trust.
The majority of patients referred for a SeHCAT scan were seen in the GI and nutrition team (GIaNT) 'consequences-of-cancertreatment' clinic. Before every GIaNT clinic appointment, patients are required to fill out a modified gastrointestinal symptom rating scale (GSRS) questionnaire, to aid clinical management. ${ }^{24}$ The GSRS used in the GIaNT clinic contains 30 questions. Of the 30 symptoms the questionnaire asks about, 13 of the GI symptoms were considered to be potentially related to bile acid malabsorption. These symptoms were urgency, abdominal bloating, faecal incontinence, flatulence, abdominal pain, steatorrhoea, nocturnal defecation, tenesmus, belching, nausea, vomiting, heartburn and borborygmi. Patients were asked to categorise the severity of each symptom as 'never', 'occasional', 'frequent' or 'causing major changes in life'.

Patients were also asked to indicate how frequently they open their bowels and, using a Bristol Stool Chart, what stool consistencies they had experienced in the previous 4 weeks. ${ }^{25}$ If a patient identified that they pass type 6 or 7 stool even intermittently, the patient was routinely offered a SeHCAT scan. We have previously shown that this policy yields an abnormal SeHCAT scan in approximately half of patients. ${ }^{18,19}$ In addition, patients were asked to rate on a scale of 1-10 their quality of life at present (where $1=$ very low and $10=$ perfect) and the severity with which their bowel symptoms affect their quality of life (where $1=$ not at all and $10=$ all the time).

Patients diagnosed with BAM were treated according to our local policy (Fig 1). ${ }^{6}$ Patients requiring a bile acid sequestrant had their dose optimised before receiving dietary advice. Patients were asked to complete a 7-day food diary for their initial consultation with a registered dietitian and then advised on a personalised low-fat dietary intervention, aiming to provide $20 \%$ of total daily energy from all types of ingested dietary fat. ${ }^{6}$ Patients attended a follow-up appointment with the dietitian 4-12 weeks later at which they brought a 7-day food diary or provided dietary recall and rated their GI symptoms again on the GSRS.

Data regarding patient demographics, previous cancer history, treatment and concomitant diagnoses that could affect their GI symptoms were also recorded.

\section{Statistical analysis}

The primary aim of this study was to evaluate the efficacy of using a low-fat diet to treat bile acid malabsorption. Patient demographics, cancer diagnosis and BAM treatment data were summarised and by SeHCAT categories using median and interquartile range (IQR) for the continuous data and counts/frequencies and percentages for the categorical data. GSRS symptom data were summarised using frequencies and percentages in the severity levels (none/mild versus moderate/ severe) groups. The difference in the paired reported frequency of symptom occurrence before and after dietary intervention as recorded on the GSRS questionnaires was assessed using McNemar's test to specify the level of statistical significance.

The Bonferroni method was used to adjust for multiple testing for the individual GSRS symptoms analysis. Based on that, only p-values $<0.002$ are considered statistically significant.

\section{Results}

This study identified 188 consecutive patients who underwent a SeHCAT scan between January 2014 and March 2016. 


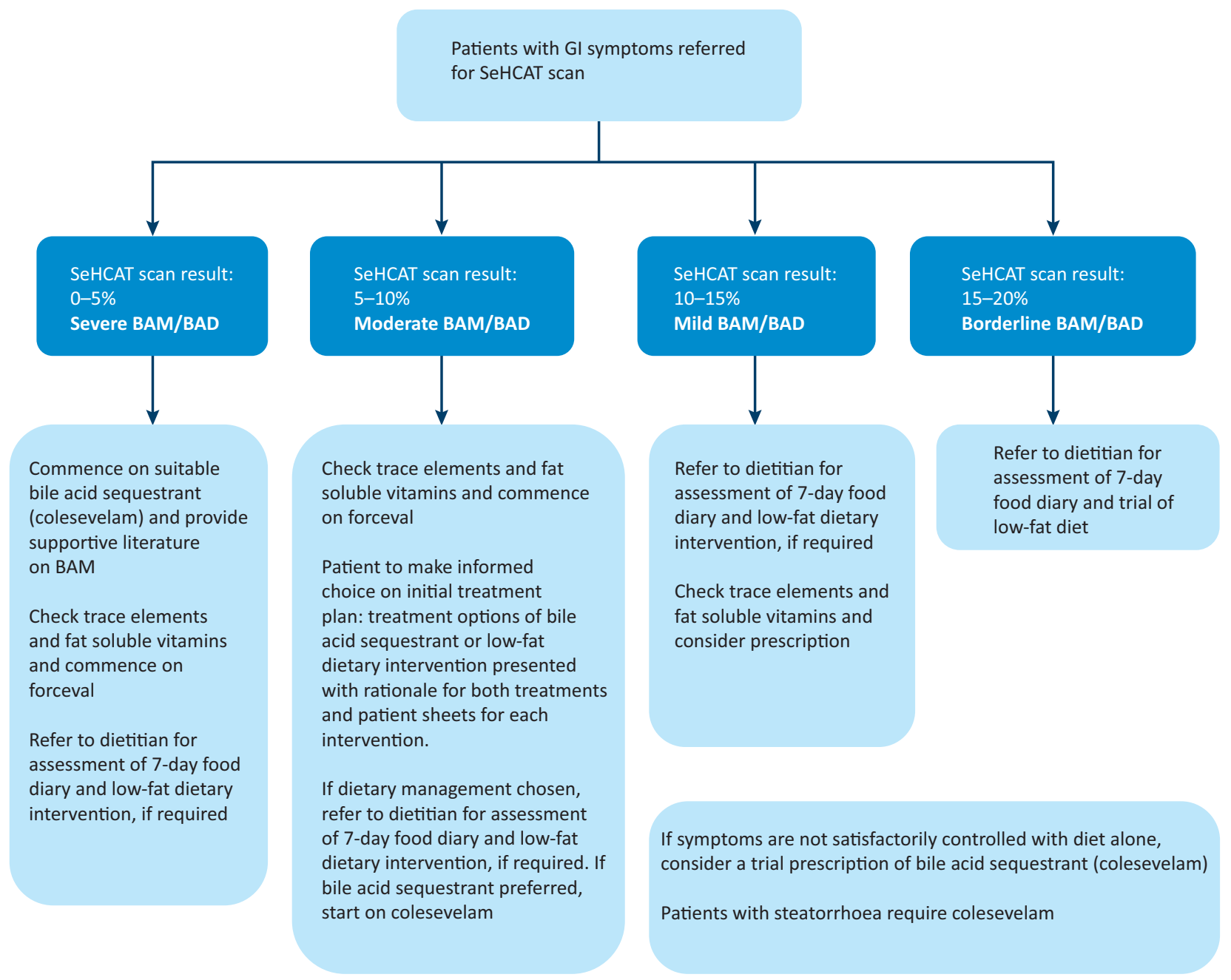

Fig 1. SeHCAT screening algorithm. Patients enrolled in this study were managed depending on their SeHCAT 7-day retention. BAD = bile acid diarrhoea; BAM = bile acid malabsorption; GI = gastrointestinal; SeHCAT = selenium -75 homocholic acid taurine.

Of these, 53 (28\%) had a 7-day retention of $15-20 \%$ (borderline), 37 (20\%) had a 7 -day retention of $10-15 \%$ (mild), 33 (18\%) had 7-day retention of 5-10\% (moderate) and $65(35 \%)$ had severe BAM (7-day retention 0-5\%).

However, not all patients had been seen in our clinic. Of those who had, a few were missing a GSRS questionnaire from relevant time points and a few did not attend follow-up. As a result, 114 patients were included in this study.

There was no significant difference in age $(\mathrm{p}=0.146)$ and numbers of patients in each SeHCAT category $(\mathrm{p}=0.07)$ between the non-evaluable and evaluable patients. The median age was similar, 61 and 64 years, respectively. However, there were fewer patients diagnosed with coexistent small intestinal bacterial overgrowth or pancreatic insufficiency in the nonevaluable group $(23(31 \%))$ compared with patients in the evaluable group (61 (54\%); $\mathrm{p}=0.002)$.

\section{Baseline characteristics}

Of the patients diagnosed with BAM, 53\% had previously undergone GI surgery and 55\% had other GI disorders, including $84 \%$ with small intestine bacterial overgrowth and $34 \%$ with pancreatic insufficiency (Table 1). At time of entry into this study, $75 \%$ of patients had been offered medication - loperamide (43\%), normacol (26\%) and colesevelam $(64 \%)$ - and were taking a stable dose.

\section{Symptom occurrence}

Table 2 shows the number of patients reporting each GI symptom and the change in each symptom after the dietary intervention for the patients overall. The change was statistically significant for abdominal pain and nocturnal defecation $(p=0.001)$. There was also a trend of improvement in most other symptoms $(\mathrm{p} \leq 0.004-0.031)$ although this was not significant at $0.2 \%$ alpha level. However, abdominal bloating $(\mathrm{p}=0.100)$, heartburn $(\mathrm{p}=0.144)$ and tenesmus $(\mathrm{p}=0.359)$ did not appear to improve much. There were no differences in response in the four separate categories of severe, moderate, mild and borderline BAM/BAD to dietary intervention (data not shown).

Fig 2 shows the stool consistency reported by the patients before and after low-fat dietary intervention, measured using the Bristol 
Table 1. Baseline characteristics of the patients included in this study

\begin{tabular}{|c|c|c|c|c|c|}
\hline \multirow{3}{*}{ Variables } & \multicolumn{5}{|c|}{ SeHCAT category } \\
\hline & Total & Severe & Moderate & Mild & Borderline \\
\hline & $n=114$ & $\begin{array}{l}(0-5 \%) \\
(n=34)\end{array}$ & $\begin{array}{l}(5-10 \%) \\
(n=19)\end{array}$ & $\begin{array}{l}(10-15 \%) \\
(n=29)\end{array}$ & $\begin{array}{l}(>15 \%) \\
(n=32)\end{array}$ \\
\hline & n (\%) & n (\%) & n (\%) & n (\%) & n (\%) \\
\hline \multicolumn{6}{|l|}{ Gender } \\
\hline Female & $65(57.0)$ & $24(37)$ & $15(23)$ & $14(22)$ & $12(18)$ \\
\hline Male & $49(43.0)$ & $10(20)$ & $4(8)$ & $15(31)$ & $20(41)$ \\
\hline \multicolumn{6}{|l|}{ Age, years } \\
\hline Median (range) & $64(23-86)$ & $61(30-84)$ & $65(23-85)$ & $63(28-80)$ & $67(40-86)$ \\
\hline Interquartile range & $55-71$ & $52-71$ & $56-69$ & $50-70$ & $58-74$ \\
\hline \multicolumn{6}{|l|}{ Weight, kg } \\
\hline Median (range) & 77 (39-127) & 71 (39-127) & $71(48-118)$ & $78(51-117))$ & $82(45-118)$ \\
\hline Interquartile range & $64-90$ & $55-84$ & $62-95$ & $65-84$ & $66-100$ \\
\hline \multicolumn{6}{|l|}{ BMI } \\
\hline Median (range) & $27(16-45)$ & $27(17-38)$ & $28(18-44)$ & 27 (19-39) & $29(16-45)$ \\
\hline Interquartile range & $23-31$ & 20-31 & $22-35$ & $24-29$ & 23-31 \\
\hline \multicolumn{6}{|l|}{ Tumour site } \\
\hline Upper GI & $10(9)$ & $2(20)$ & 0 & $3(30)$ & $5(50)$ \\
\hline Lower GI & $25(22)$ & $8(32)$ & $7(28)$ & $5(20)$ & $5(20)$ \\
\hline Urology & $30(26)$ & $8(27)$ & $2(7)$ & $8(27)$ & $12(40)$ \\
\hline Gynaecological & $31(27)$ & $11(35)$ & $5(16)$ & $8(26)$ & $7(23)$ \\
\hline Haematology & $8(7)$ & $3(38)$ & $1(12)$ & $2(25)$ & $2(25)$ \\
\hline Other ${ }^{* *}$ & $36(32)$ & $12(33)$ & $10(28)$ & $5(14)$ & $9(25)$ \\
\hline GI surgery & $60(53)$ & $22(37)$ & $11(18)$ & $13(22)$ & $14(23)$ \\
\hline Oesophago-gastric & $6(10)$ & 0 & 0 & $2(33)$ & $4(67)$ \\
\hline Pancreatic & $4(7)$ & 0 & $1(25)$ & $1(25)$ & $2(50)$ \\
\hline Biliary & $1(2)$ & $1(100)$ & 0 & 0 & 0 \\
\hline Hepatic & $5(8)$ & $1(20)$ & $2(40)$ & 0 & $2(40)$ \\
\hline Stoma & $15(25)$ & $7(47)$ & $3(20)$ & $4(27)$ & $1(7)$ \\
\hline Small bowel & $9(15)$ & $7(78)$ & $1(11)$ & $1(11)$ & 0 \\
\hline Right colon & $14(23)$ & $6(43)$ & $2(14)$ & $3(21)$ & $3(21)$ \\
\hline Left colon & 0 & 0 & 0 & 0 & 0 \\
\hline Anterior resection & $12(20)$ & $2(17)$ & $4(33)$ & $3(25)$ & $3(25)$ \\
\hline Other surgery & $26(43)$ & $7(27)$ & $6(23)$ & $7(27)$ & $6(23)$ \\
\hline Coexisting treated disorders & $61(55)$ & $19(31)$ & $9(15)$ & $18(30)$ & $15(25)$ \\
\hline SIBO & $51(84)$ & $17(33)$ & $9(18)$ & $14(27)$ & $11(22)$ \\
\hline Pancreatic insufficiency & $21(34)$ & $6(29)$ & $3(14)$ & $7(33)$ & $5(24)$ \\
\hline Prescribed medication & $86(75)$ & $32(37)$ & $17(20)$ & $18(21)$ & $19(22)$ \\
\hline Loperamide & $37(43)$ & $9(24)$ & $6(16)$ & $8(22)$ & $14(38)$ \\
\hline Normacol & $22(26)$ & $1(5)$ & $7(32)$ & $9(41)$ & $5(23)$ \\
\hline Colesevelam & $55(64)$ & $30(55)$ & $15(27)$ & $6(11)$ & $4(7)$ \\
\hline Senna & $7(8)$ & 0 & $2(29)$ & $1(14)$ & $4(57)$ \\
\hline Sodium docusate & 0 & & & & \\
\hline Lactulose & $1(1)$ & 0 & 0 & 0 & $1(100)$ \\
\hline Laxido & $1(1)$ & 0 & $1(100)$ & 0 & 0 \\
\hline Daily colesevelam & $53(46)$ & $28(53)$ & $15(28)$ & $6(11)$ & $4(8)$ \\
\hline Daily cholestyramine & 0 & & & & \\
\hline
\end{tabular}


Table 2. Patient GSRS symptom occurrence change from pre- to post-treatment assessment

\begin{tabular}{|c|c|c|c|c|c|c|}
\hline \multirow{2}{*}{$\begin{array}{l}\text { Symptom } \\
\text { Frequency of occurrence }\end{array}$} & \multirow[t]{2}{*}{$\mathbf{n}$} & \multicolumn{2}{|c|}{ Initial assessment, $\mathrm{n}(\%)$} & \multicolumn{2}{|c|}{ Follow-up, n (\%) } & \multirow{2}{*}{$\begin{array}{l}\text { McNemar } \\
\text { p-value }\end{array}$} \\
\hline & & Never/occasional & Frequent/CMCL & Never/occasional & Frequent/CMCL & \\
\hline Urgency to open bowels & 107 & $55(51)$ & $52(49)$ & $72(67)$ & $35(33)$ & 0.005 \\
\hline Abdominal bloating & 99 & $69(70)$ & $30(30)$ & $77(78)$ & $22(22)$ & 0.100 \\
\hline Faecal incontinence & 96 & $70(73)$ & $26(27)$ & $81(84)$ & $15(16)$ & 0.013 \\
\hline Flatulence & 106 & $60(57)$ & $46(43)$ & $76(72)$ & $30(28)$ & 0.005 \\
\hline Abdominal pain & 107 & $60(56)$ & $47(44)$ & $77(72)$ & $30(28)$ & 0.001 \\
\hline Steatorrhoea & 91 & $73(80)$ & $18(20)$ & $83(91)$ & $8(9)$ & 0.031 \\
\hline Nocturnal defecation & 104 & $74(71)$ & $30(29)$ & $87(84)$ & $17(16)$ & 0.001 \\
\hline Tenesmus & 104 & $65(63)$ & $39(37)$ & $70(67)$ & $34(33)$ & 0.359 \\
\hline Belching & 107 & $84(79)$ & $23(21)$ & $96(90)$ & $11(10)$ & 0.008 \\
\hline Nausea & 106 & $84(79)$ & $22(21)$ & $94(89)$ & $12(11)$ & 0.021 \\
\hline Vomiting & 107 & $97(91)$ & $10(9)$ & $103(96)$ & $4(4)$ & 0.031 \\
\hline Heartburn & 107 & $89(83)$ & 18 (17) & $96(90)$ & $11(10)$ & 0.144 \\
\hline Borborygmi & 106 & $65(61)$ & $41(39)$ & $81(76)$ & $25(24)$ & 0.004 \\
\hline
\end{tabular}

Stool Chart. The most frequently reported stool consistency changed from stool type 5 at initial assessment to type 4 posttreatment. In addition there was a $40 \%$ drop in patients reporting type 7 stool and a 17\% decrease in patients reporting type 6 stool. Fig 3 shows the frequency of bowel movements reported by patients, before and after low-fat dietary intervention. The frequency of bowel movements remained consistent at 2-3 times per day; however, there was a reported $32 \%$ decrease in 7 or more bowel movements a day and a 30\% decrease in 4-6 bowel movements a day after dietetic intervention.

Fig 4 and Fig 5 express the change in the patients' perceived quality of life and the severity to which their bowel symptoms affected their quality of life, before and after treatment. The median score for quality of life improved from 6 to 7 (where $1=$ very low and $10=$ perfect $)$. The median score for the severity

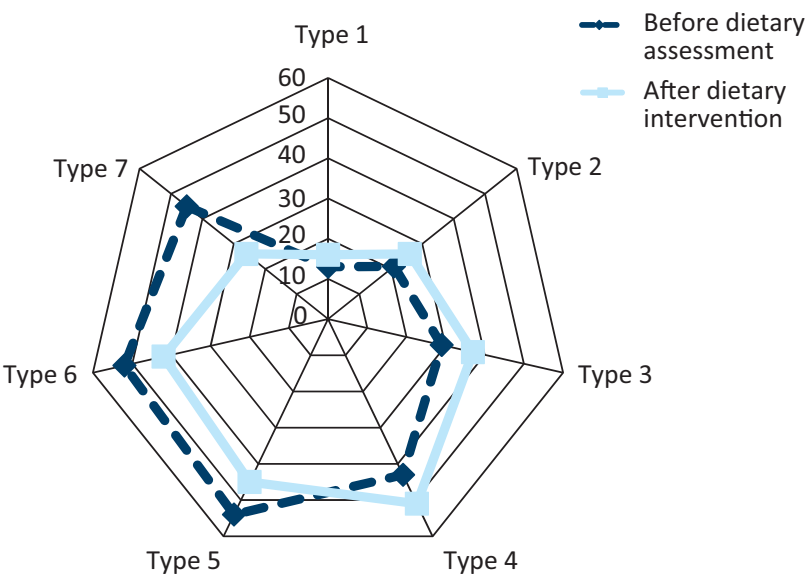

Fig 2. Stool consistency before and after dietary advice, as recorded on a Bristol Stool Chart. Stool type is indicated 1-7 and the percentage of affected people is shown 0-60\% for each stool type.

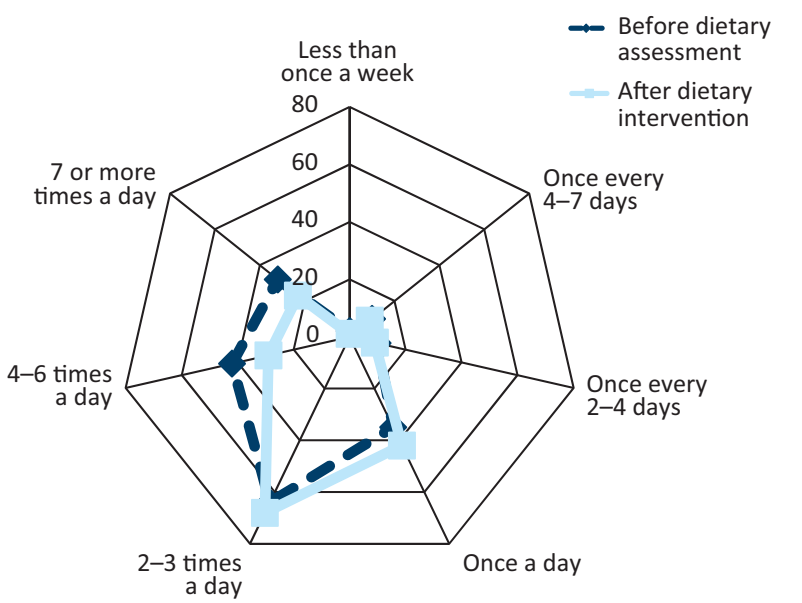

Fig 3. Stool frequency before and after dietary advice, as recorded on a Bristol Stool Chart. Stool frequency is indicated as less than once a week through to seven times per day and the percentage of affected people is shown $0-70 \%$ for each stool type.

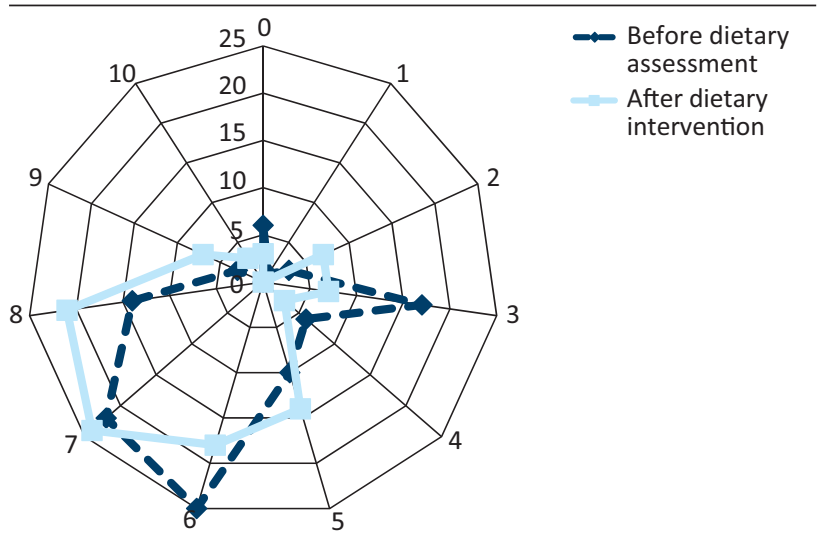

Fig 4. Patient rating for their overall quality of life before and after the dietetic intervention. Patients completed a visual analogue scale to assess their quality of life, where 0 signifies a low score and 10 a perfect quality of life. 


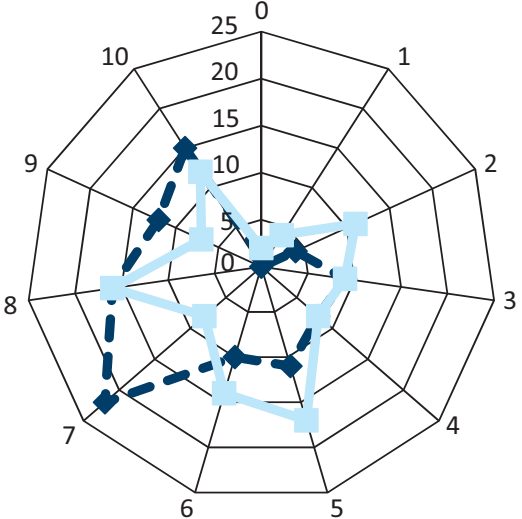

Fig 5. Patient rating for their bowel-specific quality of life before and after the dietetic intervention. In contrast to the overall quality of life scale, for bowel function, 0 signifies no effect of bowel symptoms on quality of life while 10 signifies terrible effects.

to which their bowel symptoms affected their quality of life improved from 7 to $6(1=$ not at all, $10=$ all the time $)$.

\section{Discussion}

This evaluation shows that after a low-fat dietary intervention the frequency with which patients with a SeHCAT scan retention of $<20 \%$ experienced GI symptoms decreased, with a significant reduction in abdominal pain and nocturnal defecation and a trend towards statistically significant improvement for most other symptoms. These improvements were seen both in patients on no bile acid sequestrants and those on a stable dose of sequestrant but still with residual symptoms.

While guidelines generally consider a SeHCAT scan retention of $>15 \%$ normal, it has been shown that if a scan is repeated twice in the same patient, the values, while close, are not always identical. ${ }^{9}$ Consequently, it is possible that some patients with borderline BAM/BAD may in fact have more severe malabsorption. Symptoms will also depend on the amount of fat ingested, something rarely considered by clinicians. Therefore, an absolute cut-off value defining an abnormal SeHCAT scan is not rational. This study has shown that patients with borderline BAM according to a SeHCAT scan do experience GI symptoms, which sometimes can be improved with suitable interventions.

This was not a blinded study and patients acted as their own controls. Some patients had to be excluded as they did not return for follow-up or the GSRS questionnaire from their follow-up visit was not saved to our electronic patient record. The group of patients we see are complex and very frequently have multiple GI diagnoses contributing to their symptoms. ${ }^{26}$ Other diagnoses were excluded or treated using the GIaNT management algorithm. ${ }^{27}$ One third of patients also had a degree of pancreatic insufficiency, so dietary fat intake may have impacted on these patients' symptoms although patients were established on all appropriate medication before dietary intervention; ie dietary intervention is the last therapeutic intervention in our clinic. We performed quite a number of statistical analyses and as such some of our positive findings may have occurred by chance although we have corrected for multiple testing. While all of these factors have the potential to bias the results, our overall findings are consistent with much smaller studies, some from many years ago. ${ }^{21,23,28-30}$ Our findings are also consistent with a smaller study from our unit in a separate population of 40 patients assessed using a different numerical symptom scoring tool published in $2015 .^{6}$

Dietary fat intake was assessed via 7-day food diaries, which has been shown to have far greater accuracy than a 24-hour dietary recall. ${ }^{31}$ All food diaries were analysed by one of two dietitians. Previous studies have shown that dietary fat intake limited to $20 \%$ of daily calorie intake can be maintained by patients long term. ${ }^{6}$ The use of low-fat dietary intervention in the management of BAM has been overlooked in national guidelines. ${ }^{6}$ The use of such dietary intervention can have a significant impact on GI symptoms and quality of life also improved as scored in this study. The impact on the health economy of effectively managing the condition in this way is likely to be substantial.

\section{Conclusions}

This study suggests that for patients with a SeHCAT retention of $<20 \%$, management of GI symptoms with the use of a low-fat diet leads to a clinically significant improvement in symptoms and should be a routine part of the therapeutic armamentarium in this patient group. Further studies are warranted to determine the degree of fat restriction that is effective and the long-term sustainability of this intervention by patients.

\section{Conflicts of interest}

HJNA has chaired advisory boards for GE, manufacturers of SeHCAT, and for Sanofi Aventis and Genzyme, manufacturers of colesevelam. HJNA has also received honoraria as a speaker and consultant for these companies. The Royal Marsden Hospital has received free SeHCAT capsules for a separate study for which HJNA is the CI.

\section{Author contributions}

AJ developed the study idea, wrote the study protocol, collected the data, analysed the data, wrote the first draft of the manuscript and approved the final draft. AL collected the data, entered the data into the study database and approved the final draft. MK analysed the data. AM treated the patients, collected the data and approved the final draft. CG treated the patients, collected the data and approved the final draft. SV developed the study idea, treated the patients and approved the final draft. CS developed the study idea, treated the patients, collected the data and approved the final draft. JA conceived and helped develop the study idea, treated the patients, drafted the final version of the paper, and is the guarantor of the study.

\section{Acknowledgements}

We acknowledge support from the National Institute for Health Research Royal Marsden Biomedical Research Centre.

\section{References}

1 Brand SJ, Schmidt WE. Gastrointestinal Hornones. In: Yamada T (ed). Textbook of gastroenterology. Philadelphia: JB Lippincott Company,1995:25-71.

2 Walters JRF. Bile acid diarrhoea and FGF19: new views on diagnosis, pathogenesis and therapy. Nat Rev Gastroenterol Hepatol 2014;11:426-34. 
3 Chiang JY. Bile acids: regulation of synthesis. J Lipid Res 2009;50:1955-66.

4 Wedlake L, Thomas K, Lalji A, Anagnostopoulos C, Andreyev HJN. Effectiveness and tolerability of colesevelam hydrochloride for bile-acid malabsorption in patients with cancer: a retrospective chart review and patient questionnaire. Clin Ther 2009;31:2549-58.

5 Pattni S, Walters JR. Recent advances in the understanding of bile acid malabsorption. Br Med Bull 2009;92:79-93.

6 Watson L, Lalji A, Bodla S et al. Management of bile acid malabsorption using low fat dietary interventions: a useful strategy applicable to some patients with diarrhoea predominant irritable bowel syndrome? Clin Med 2015;15:536-40.

7 Walters JR, Tasleem AM, Omer OS et al. A new mechanism for bile acid diarrhea: defective feedback inhibition of bile acid biosynthesis. Clin Gastroenterol Hepatol 2009;7:1189-94.

8 Merrick MV, Eastwood MA, Ford MJ. Is bile acid malabsorption underdiagnosed? An evaluation of accuracy of diagnosis by measurement of SeHCAT retention. Br Med J 1985;290:665-8.

9 Bajor A, Kilander A, Sjövall H, Rudling M, Ung KA. The bile acid turnover rate assessed with the (75)SeHCAT test is stable in chronic diarrhoea but slightly decreased in healthy subjects after a long period of time. Dig Dis Sci 2008;53:2935-40.

10 Wedlake L, A'Hern R, Russell D et al. Systematic review: the prevalence of idiopathic bile acid malabsorption as diagnosed by SeHCAT scanning in patients with diarrhoea-predominant irritable bowel syndrome. Aliment Pharmacol Ther 2009;30:707-17.

11 al-Hadrani A, Lavelle-Jones M, Kennedy $\mathrm{N}$ et al. Bile acid malabsorption in patients with post-vagotomy diarrhoea. Ann Chir Gynaecol 1992;81:351-3.

12 Sciarretta G, Furno A, Mazzoni M, Malaguti P. Postcholecystectomy diarrhea: evidence of bile acid malabsorption assessed by SeHCAT test. Am J Gastroenterol 1992;87:1852-4.

13 Raju GS, Dawson B, Bardhan KD. Bile acid malabsorption associated with Graves' disease. J Clin Gastroenterol 1994;19:54-6.

14 Cramp ME, Hing MC, Marriott DJ, Freund J, Cooper DA. Bile acid malabsorption in HIV infected patients with chronic diarrhoea. Aust N Z J Med 1996;26:368-71.

15 Dutta SK, Anand K, Gadacz TR. Bile salt malabsorption in pancreatic insufficiency secondary to alcoholic pancreatitis. Gastroenterology 1986;91:1243-9.

16 Ung KA, Gillberg R, Kilander A, Abrahamsson H. Role of bile acids and bile acid binding agents in patients with collagenous colitis. Gut 2000;46:170-5.

17 Pawlyn C, Khan MS, Muls A et al. Lenalidomide-induced diarrhea in patients with myeloma is caused by bile acid malabsorption that responds to treatment. Blood 2014;124:2467-8.

18 Gupta A, Muls AC, Lalji A et al. Outcomes from treating bile acid malabsorption using a multidisciplinary approach. Support Care Cancer 2015;23:2881-90.

19 Phillips F, Muls ACG, Lalji A, Andreyev HJN. Are bile acid malabsorption and bile acid diarrhoea important causes of diarrhoea complicating cancer therapy? Colorectal Dis 2015;17:730-4.
20 Hassan B, Lightowlers S, Matakidou A et al. Bile acid malabsorption is a common, reversible cause of diarrhoea in patients on tyrosine kinase inhibitors for metastatic renal cell carcinoma [abstract]. Liverpool: 2015 NCRI Cancer Conference, 2015: abstract B60. http://abstracts.ncri.org.uk/abstract/bile-acid-malabsorption-is-acommon-reversible-cause-of-diarrhoea-in-patients-on-tyrosinekinase-inhibitors-for-metastatic-renal-cell-carcinoma-2/ [Accessed 11 July 2017].

21 Bosaeus I, Andersson H, Nystrom C. Effect of a low-fat diet on bile salt excretion and diarrhoea in the gastrointestinal radiation syndrome. Acta Radiol Oncol Radiat Phys Biol 1979;18:460-4.

22 Nelson LM, Carmichael HA, Russell RI, Atherton ST. Use of an elemental diet (Vivonex) in the management of bile acid-induced diarrhoea. Gut 1977;18:792-4.

23 Danielsson A, Nyhlin H, Persson $\mathrm{H}$ et al. Chronic diarrhoea after radiotherapy for gynaecological cancer: occurrence and aetiology. Gut 1991;32:1180-7.

24 Svedlund J, Sjödin I, Dotevall G. GSRS-a clinical rating scale for gastrointestinal symptoms in patients with irritable bowel syndrome and peptic ulcer disease. Dig Dis Sci 1988;33:129-34.

25 Lewis SJ, Heaton KW. Stool form scale as a useful guide to intestinal transit time. Scand J Gastroenterol 1997;32:920-4.

26 Muls AC, Lalji A, Marshall C et al. The holistic management of consequences of cancer treatment by a gastrointestinal and nutrition team: a financially viable approach to an enormous problem? Clin Med 2016;16:240-6.

27 Andreyev HJN, Benton BE, Lalji A et al. Algorithm-based management of patients with gastrointestinal symptoms in patients after pelvic radiation treatment (ORBIT): a randomised controlled trial. Lancet 2013;382:2084-92.

28 Koga T, Nishida T, Miwa $\mathrm{H}$ et al. Effects of dietary butter fat on fecal bile acid excretion in patients with Crohn's disease on elemental diet. Dig Dis Sci 1984;29:994-9.

29 Reddy BS, Engle A, Simi B et al. Effect of low-fat, high-carbohydrate, high-fiber diet on fecal bile acids and neutral sterols. Prev Med 1988;17:432-9.

30 Costarelli V, Sanders TA. Acute effects of dietary fat composition on postprandial plasma bile acid and cholecystokinin concentrations in healthy premenopausal women. Br J Nutr 2001;86:471-7.

31 Bingham SA, Gill C, Welch A et al. Validation of dietary assessment methods in the UK arm of EPIC using weighed records, and 24-hour urinary nitrogen and potassium and serum vitamin $\mathrm{C}$ and carotenoids as biomarkers. Int J Epidemiol 1997;26(Suppl 1):S137-51.

Address for correspondence: Dr HJN Andreyev, GI Unit, United Lincolnshire Hospitals NHS Trust, Lincoln County Hospital, Greetwell Road, Lincoln LN2 5QY, UK.

Email: j@andreyev.demon.co.uk 